\title{
Hydroxychloroquine: A review of its effects on viral replication based on current literature
}

\author{
Medha Ghose MBBS, Maehali Patel BA, Kenneth Nugent MD
}

\begin{abstract}
Quinine has been used for centuries as a drug for malaria treatment. Chloroquine (CQ) and its 4-aminoquinoline analog hydroxychloroquine (HCQ) have been used during the last two centuries for the treatment and prophylaxis of malaria. Since the inception of COVID-19 in Wuhan, China, in late December 2019, physicians and researchers have tried to use old drugs to treat this pandemic. This review discusses possible modes of action of $C Q$ and $H C Q$ in vitro that might treat this viral infection. The diverse cellular, immunological, and antiviral mechanisms reported in the literature suggest that $C Q$ and HCQ might be useful drugs against COVID-19. Both drugs increase the $\mathrm{pH}$ in intracellular organelles, which limits viral replication. However, randomized controlled trials have not found any benefit as either for prophylaxis or treatment of COVID-19. In addition, fatal adverse events associated with these drugs have resulted in the issuance of precautionary measures when recommending these drugs in COVID-19 patients. The search for other innovative drugs to cure COVID-19 and an expedited approval for a vaccine are ongoing at present.
\end{abstract}

Keywords: hydroxychloroquine, chloroquine, COVID 19, antiviral effects, immune response

\section{INTRODUCTION}

Chloroquine (CQ) and its analog, hydroxychloroquine $(\mathrm{HCQ})$, are well-known drugs that have been used as antimalarial drugs for the last century. Their parent compound, quinine, was isolated from the bark of the cinchona tree in 1820 by French researchers Pierre Joseph Pelletier and Joseph Bienaimé Caventou. The earliest reported use dates back to the 1630 s in Peru when it is claimed that the Countess of Chinchon, from whom the tree's name was derived, was treated with tree bark extract for an unknown febrile illness that was likely malaria. Around 1894, it

Corresponding author: Medha Ghose Contact Information: Medhaghose1692@gmail.com DOI: $10.12746 /$ swrccc.v8i36.777 started to gain recognition for its benefit in rheumatologic and autoimmune diseases when Payne reported its effectiveness in treating cutaneous lupus. ${ }^{1}$ The alkaloids derived from the cinchona tree bark, including $\mathrm{CQ}$ and other 4-aminoquinolines, started being investigated at the beginning of 1943 as a part of the antimalarial research program in the United States during World War II. Researchers also observed improvement in rashes and inflammatory joint pain in soldiers, and the first trial of this antimalarial drug as a treatment option for systemic lupus erythematosus was conducted. ${ }^{2}$ Since then, HCQ has been successfully used to treat rheumatologic syndromes (systemic lupus erythematosus, ${ }^{3}$ antiphospholipid antibody syndrome ${ }^{4}$ rheumatoid arthritis, ${ }^{5}$ Sjogren's syndrome ${ }^{6}$ ), infectious diseases (HIV, Q fever, Whipple's disease, fungal infections), and multiple neoplasms (breast cancer, colon cancer, glioblastoma multiforme).$^{7-11}$ 
This broad use pattern would suggest that at minimum these drugs are relatively safe.

Since the outbreak of novel severe acute respiratory syndrome coronavirus 2 (SARS-CoV-2) (COVID19), first reported in Wuhan, China, in December 2019, over 26 million people have been infected with over 870,000 deaths as of September 3, 2020. ${ }^{12}$ COVID-19 is an acute disease that can be severe and fatal if not timely managed. Severe COVID-19 disease is characterized by extensive alveolar damage with acute hypoxemic respiratory failure and death. ${ }^{13}$ Beginning in April 2020 , autopsy studies have provided important information about this disease..$^{14}$ Two extensive studies conducted by Swiss and German researchers that included 21 and 12 COVID patients, respectively, showed that the primary cause of death was respiratory failure with exudative alveolar damage, massive capillary congestion with concomitant microthrombi despite anticoagulation, and superimposed bronchopneumonia in almost half of the cases..$^{15} \mathrm{~A}$ Phase II Clinical Trial to Evaluate the Recombinant Vaccine for COVID-19 (Adenovirus Vector) is being conducted by Chinese researchers; vaccines, of course, have the potential to prevent COVID-19 spread among healthy adults aged 18 years or older. ${ }^{16}$ However, no specific antiviral drug has been confirmed to be effective in treating COVID-19, and given the current circumstances, many existing drugs, including $\mathrm{HCQ}$, are being considered for repurposing against Coronavirus 2. Scientific data accumulated over the years have highlighted antiviral properties that could be relevant in COVID-19 treatment.

\section{HISTORICAL DEVELOPMENT OF HYDROXYCHLOROQUINE AND ITS ANALOGS}

It is said that the Incas in Peru used the cinchona tree's bark to cure the countess of Chinchon from a mysterious febrile illness in $1630 .{ }^{17}$ In 1891, Paul Ehrlich and colleagues demonstrated the uptake of the dye methylene blue by malarial parasites and later used that knowledge to replace the methyl group with a basic chain to develop pamaquine, a drug with antimalarial effects. Similar chemical modifications were made subsequently to produce compounds, such as chloroquine, quinacrine, sontoquine, and primaquine. Chloroquine, brand name Resochin, was used as an antimalarial drug at the beginning of 1943, during World War II, as a part of the extensive cooperative program of antimalarial research in the United States. Investigators also observed improvement in rashes and inflammatory joint pain in soldiers, and the first trial of antimalarial drug for the treatment of systemic lupus erythematosus (SLE) was conducted. ${ }^{2}$

\section{General molecular mechanism of action}

Chloroquine and hydroxychloroquine, in unprotonated forms, generally act as weak bases that affect acidic enzyme containing vesicle organelles, leading to dysfunction. Upon entering the intracellular compartment of the vesicle organelles, such as endosomes, Golgi vesicles, and lysosomes, these drugs become protonated by following a HendersonHasselbach reaction. This phenomenon increases the $\mathrm{pH}$ of the lysosomal and trans-Golgi network vesicles, leading to multiple effects, including the inhibition of post-translational modification of newly synthesized proteins, impairment of endosomal release of iron from ferrated transferrin, which leads to decreased intracellular iron concentration, and reduced DNA replication and gene expression. These drug effects are characteristic of lysosomotropic agents. ${ }^{18,19}$

Increases in the $\mathrm{pH}$ of the lysosomes and transGolgi network by $\mathrm{CQ}$ and $\mathrm{HCQ}$ affect several cellular processes, including phagocytosis, exosome release, and phagolysosomal fusion. Prevention of phagolysosomal fusion leads to decreased antigen processing by antigen-presenting cells, such as plasmacytoid dendritic cells and $\mathrm{B}$ cells, thus causing failure of major histocompatibility complex (MHC) class II-mediated autoantigen presentation to $\mathrm{T}$ cells. ${ }^{20} \mathrm{As}$ a result, $\mathrm{T}$ cell activation, differentiation, and production of co-stimulatory proteins (e.g., CD154 on CD4+ T cells) and $T$ cells and $B$ cells cytokines are inhibited. ${ }^{21}$ In addition, HCQ also interrupts the binding between Toll-like receptors (TLR7 and TLR9) and their RNA/DNA ligands and prevents the interaction between cytosolic DNA and the nucleic acid sensor cyclic GMP-AMP synthase, thus suppressing TLR signaling and subsequent activation and production of the inflammatory cytokines IL-1, type I interferons, and TNF, also known as the STING pathway. ${ }^{22-24}$ 


\section{ANTIVIRAL MECHANISM OF ACTION}

Chloroquine and hydroxychloroquine are multifunctional drugs that, along with their antirheumatic and antineoplastic activity, also have diverse antiviral activity against both RNA and DNA viruses. They have broad-spectrum activity against enveloped viruses and prevent endosome mediated viral entry and post-translational modification. Usually, upon entering the target cells by endocytosis, some viruses enter the lysosome and release their infectious nucleic acid in the low pH environment. However, $\mathrm{CQ}$ and $\mathrm{HCQ}$ can inhibit the entry of these viruses by increasing the $\mathrm{pH}$ of the lysosome. For example, hepatitis A virus is affected by this mechanism as $C Q$ and $H C Q$ prevents its replication by inhibiting its uncoating. ${ }^{25}$

Chloroquine and hydroxychloroquine also prevent post-translational modifications such as glycosylation of some enveloped viruses within the endoplasmic and TGN vesicles. Since viral enzyme activity depends on maintaining low $\mathrm{pH}$ within the organelles, $\mathrm{CQ}$ and $\mathrm{HCQ}$ prevent their replication by inhibiting the activity of these enzymes through increases in the $\mathrm{pH}$ in these organelles. ${ }^{26}$ Prevention of glycosylation also leads to non-infectious viral particles and reduced total vital particle production that eventually reduces viral infectivity. This effect has been shown in studies with the avian reticuloendotheliosis virus REV-A and with HIV-1. ${ }^{27}$ Chloroquine and hydroxychloroquine also prevent the proteolytic processing of viral prM protein, which affects the viral replication cycle in a particular viral family called the Flaviviridae. ${ }^{28}$

Chloroquine and hydroxychloroquine have shown promising antiviral activity in vitro against other RNA viruses, such as poliovirus, ${ }^{29}$ rabies virus, ${ }^{30}$ hepatitis C virus, ${ }^{31}$ influenza $A$ and $B$ viruses, ${ }^{32-35}$ influenza A H5N1 virus, ${ }^{36}$ Chikungunya virus, ${ }^{37}$ Dengue virus, ${ }^{38}$ Zika virus, ${ }^{39}$ Lassa virus, ${ }^{40}$ Hendra and Nipah viruses, ${ }^{41-42}$ Ebola virus, ${ }^{43}$ Crimean-Congo hemorrhagic fever virus, ${ }^{44}$ and DNA viruses, such as herpes simplex virus ${ }^{45}$ and hepatitis $B$ virus. ${ }^{46}$ However, in vivo clinical trials sometimes failed to produce satisfactory results perhaps secondary to the disease process, the concentration of chloroquine used, treatment duration, and time of drug administration. ${ }^{47}$

\section{IMMUNOMODULATORY ACTION}

Intracellular accumulation of lysosomotropic weak bases, such as $C Q$ and $H C Q$, especially in lymphocytes and macrophages, mediates the immunomodulatory effects that have led to their clinical use in inflammatory conditions, including rheumatoid arthritis, lupus erythematosus, and sarcoidosis. Chloroquine and hydroxychloroquine reduce the release of pro-inflammatory cytokines, specifically tumor necrosis factor-alpha (TNF- $\alpha$ ), from human peripheral blood mononuclear cells and human whole blood in a dose-dependent fashion. Several mechanisms may contribute to this, including disruption of iron homeostasis, interruption of phospholipase A2 induced expression of TNF $\alpha$, profound inhibition of prohormone processing of proTNF- $\alpha$ to a mature form, and reduction in TNF-alpha mRNA expression by a non-lysosomotropic mechanism. ${ }^{48-51}$ Surface expression of TNF-alpha is also reduced by $C Q$ and $H C Q$. These processes subsequently result in impairment of pro-inflammatory signaling and mitigation of cytokine production, such as IL-1 and TNF, which have a significant role in cytokine storm syndrome that may be attenuated by $C Q$ and $\mathrm{HCQ}$. In summary, CQ and HCQ could limit the inflammatory response associated with this viral infection, but this effect could also limit the host defenses needed to clear the virus.

\section{ANTIVIRAL ACTION AGAINST CORONAVIRUS INFECTION}

Inhibition of post-translational glycosylation and the subsequent reduction of binding and fusion of SARS-CoV-1 to the host cell receptor angiotensin-converting enzyme 2 (ACE2) is an important antiviral effect of CQ and HCQ treatment in SARS-CoV-1 infections. SARS-CoV-2 has been thought to utilize the same mechanism for cellular entry. ${ }^{52}$ Cleavage of Spike (S) protein in SARS-CoV-2 by CQ and HCQ in autophagosomes has also been reported. ${ }^{53}$

The efficacy of $\mathrm{CQ}$ and $\mathrm{HCQ}$ in vitro against other respiratory viruses, like human coronavirus $\mathrm{HCoV}-\mathrm{O} 43$ and orthomyxoviruses, has been reported. Respiratory viruses like influenza virus and coronavirus enter cells 
by binding to sialic acid-linked ganglioside receptors in the respiratory tract. Sialic acid ganglioside and glycoproteins have been observed as cell-surface attachment factors responsible for high transmissibility. ${ }^{54}$ In addition, the transmembrane serine protease 2 (TMPRSS2) and extracellular matrix metalloproteinase inducer CD147, also called basigin, probably facilitate the entry of SARS-CoV into human cells. However, HCQ was found ineffective in blocking TMPRSS2. ${ }^{55,56}$

\section{Clinical tRIals on CQ/HCQ against SARS-CoV-2}

Initially $\mathrm{CQ}$ and $\mathrm{HCQ}$ were considered for the treatment of COVID-19 based on preclinical in vitro data showing antiviral properties against SARSCoV-2, even though there were no reports of their use for prevention or treatment.

Since March 2020, multiple clinical trials have been conducted to determine the efficacy of $C Q$ and $\mathrm{HCQ}$ in treating COVID-19. Gautret et al. treated 20 patients in a non-randomized clinical trial with HCQ $(200 \mathrm{mg}$ three times a day for 10 days) or HCQ plus azithromycin (AZM, $500 \mathrm{mg}$ on day 1 followed by $250 \mathrm{mg} /$ day for the next 4 days) and did serial viral cultures on nasopharyngeal secretions. ${ }^{57}$ Seventy percent of the patients had negative viral studies by day six; $12.5 \%$ with patients in the untreated group had negative studies $(P=0.001)$. These investigators suggested starting $\mathrm{HCQ}$ treatment well before the patients develop Acute Respiratory Distress Syndrome (ARDS) associated with cytokine storm. ${ }^{57}$ Huang et al. randomized 22 patients into the 2 treatment arms. ${ }^{58}$ Ten patients received chloroquine phosphate, and 12 patients received lopinavir/ritonavir. All patients in the chloroquine group became RT-PCR negative by day 14 ; 11 of the patients on lopinavir/ritonavir became negative by day 14 . The CT images improved faster in the patients on chloroquine phosphate, and these patients were discharged from the hospital earlier. Gao et al. reviewed ongoing trials in China with a chloroquine phosphate in February $2020 . .^{59}$ These trials included 100 patients and demonstrated that chloroquine phosphate works better than control treatment in inhibiting "exacerbation of pneumonia," improving lung imaging "findings," promoting virus negative conversion, and shortening the disease course. Rana and Dulal summarized trials using chloroquine in the treatment of COVID-19 from information available in various registries as of March 16, 2020. There were 21 trials listed, but no information was available on outcomes. ${ }^{60}$

A large retrospective study was done in the United States with 368 patients in Veterans Health Administration Medical Centers. ${ }^{61}$ These patients were followed over a period of 14 days with one group receiving $\mathrm{HCQ}$ or $\mathrm{HCQ}+\mathrm{AZM}$ combination and the other group receiving conventional supportive care for COVID-19. This study focused on two primary outcomes, namely mechanical ventilation requirement and death. At the end of the trial, the authors concluded that $\mathrm{HCQ}$ alone or in combination with AZM showed no benefit with a higher mortality rate in patients treated with $\mathrm{HCQ}$ alone (27.8\%) and the combination of HCQ+AZM (22.1\%) compared to the group receiving no drug treatment (11.4\%). ${ }^{61}$ In April 2020, the use of optimal HCQ dose for SARS-CoV-2 was encouraged, which required a longer duration of treatment, along with administration of loading dose $(800 \mathrm{mg}$ twice a day for 1-2 days followed by $400 \mathrm{mg}$ bid maintenance dose). ${ }^{62} \mathrm{~A}$ recent retrospective observational study with 2,541 patients from the Henry Ford Health System in Southeast Michigan reported that $13 \%$ of the cases treated with HCQ alone (1202 patients) resulted in fatality as opposed to a $26.4 \%$ fatality rate in cases not treated with hydroxychloroquine (409 patients). Researchers noted a $66 \%$ hazard ratio reduction upon HCQ administration alone, and $71 \%$ with hydroxychloroquine plus azithromycin compared to treatment using neither drug $(p<0.001){ }^{63}$

Since these early reports on the use of either hydroxychloroquine or chloroquine were published, several randomized control trials have been completed. Boulware et al. conducted a randomized, double blind, control-placebo controlled trial in the United States and Canada to determine whether hydroxychloroquine can prevent symptomatic infection after SARS-CoV-2 exposure. ${ }^{64}$ Eight hundred twenty-one asymptomatic participants were enrolled and received either hydroxychloroquine for 5 days or placebo for 5 days. Forty-nine of 414 participants $(11.8 \%)$ receiving hydroxychloroquine and 58 of 404 participants (14.3\%) 
receiving placebo developed a new illness compatible with COVID-19. This difference was not significant. Mitja and co-investigators studied 293 non-hospitalized patients with mild to moderate COVID-19. ${ }^{65}$ These patients had symptoms for fewer than 5 days and were randomized to receive hydroxychloroquine for 6 days or no antiviral treatment. There was no significant difference in the mean viral load at baseline, at 3 days, or at 7 days between the 2 groups. Treatment did not reduce the risk of hospitalization, which was $7.1 \%$ in control patients and $5.9 \%$ in hydroxychloroquine treated patients. Finally, patients receiving hydroxychloroquine did not have a reduced time to complete resolution of symptoms. Cavalcanti et al. randomized 667 hospitalized patients to hydroxychloroquine for 7 days, hydroxychloroquine and azithromycin for 7 days, or standard care. ${ }^{66}$ These patients were either on no supplemental oxygen or less than $4 \mathrm{~L} /$ min supplemental oxygen. The primary outcome was a symptom score at 15 days. There was no difference in outcomes among these 3 groups. Pathak and colleagues performed a systematic review and meta-analysis of 7 studies reporting outcomes in COVID-19 patients treated with hydroxychloroquine. ${ }^{67}$ This study population included 4,984 patients. Approximately one-third received hydroxychloroquine and two-thirds received standard care. The odds ratio for a favorable outcome with hydroxychloroquine was $1.11(95 \% \mathrm{Cl}$ : 0.72-1.69, $\mathrm{P}=0.20)$. Consequently, this analysis indicated that hydroxychloroquine has no benefit in patients with mild to moderate COVID-19 disease. In summary, these randomized controlled trials demonstrated that hydroxychloroquine does not prevent symptomatic infection following exposure and does not change the course of mild to moderate COVID-19.

\section{ADVERSE EFFECTS OF HYDROXYCHLOROQUINE}

Several studies have reported severe QT prolongation and cardiac arrhythmias in patients treated with $\mathrm{CQ} / \mathrm{HCQ}$. A meta-analysis by Tleyjeh et al., including 19 studies with 5,652 patients, reported two episodes of torsades de pointes in 2719 patients treated with $\mathrm{CQ} / \mathrm{HCQ}$. The pooled incidence of this meta-analysis reported torsades de pointes or cardiac arrest or ventricular tachycardia in 3 out of 1000 patients. QT prolongation from the baseline was estimated $7 \%$ from 12 studies of 2,008 patients. ${ }^{68}$ Garci-Cremades et al. explained the effect of high dose HCQ $(>600 \mathrm{mg}$ b.i.d for $\geq 5$ days) on prolonging QTc value form baseline. ${ }^{62} \mathrm{~A}$ study with 84 SARS-CoV-2 patients from New York University's Langone Medical Center reported that $30 \%$ of the patients had their QTc increase by $>40 \mathrm{~ms}$, and $11 \%$ of patients had their QTc increased to $>500 \mathrm{~ms}^{69}$ However, a recent multi-center retrospective observational study from the Henry Ford Health System (HFHS) documented zero case of torsades de pointes in the 2,132 patients treated with $\mathrm{HCQ}$ alone, $\mathrm{HCQ}+\mathrm{AZM}$, or AZM alone. ${ }^{63}$

Chloroquine and hydroxychloroquine are associated with other adverse effects, including vomiting and diarrhea. ${ }^{70}$ Hydroxychloroquine is associated with important neurological side effects, including muscular weakness, diplopia, dyskinesia, seizures, and myasthenic syndrome with short-term use. Psychiatric side effects include sleeplessness, agitation, psychosis, depression, anxiety, and aggressiveness; confusion has also been reported, starting within a few days after the beginning of treatment. ${ }^{71}$ Chloroquine has a worse safety profile than $\mathrm{HCQ}$, as a one-time dose of $20 \mathrm{mg} /$ $\mathrm{kg} \mathrm{CQ}$ can be toxic, and doses above $30 \mathrm{mg} / \mathrm{kg} \mathrm{CQ}$ are considered fatal. ${ }^{72} \mathrm{~A}$ recent report also suggests that methemoglobinemia can occur in patients treated with COVID-19, including patients not having previous history of G6PD deficiency. ${ }^{73}$ Long term exposure can cause severe side effects, such as retinopathy, "bull's eye" maculopathy, diametric defects in the retina, and cardiomyopathy. ${ }^{74}$ In contrast to CQ, HCQ has a lower level of tissue accumulation, which results in fewer adverse events. Chloroquine is contraindicated in pregnant patients due to its teratogenic effects, while $H C Q$ is recommended for pregnant patients with autoimmune diseases, such as SLE due to its capacity to prevent heart block. ${ }^{75}$ Based on the recent clinical data, in July 2020, the FDA issued a caution against CQ and HCQ's being used to treat hospitalized patients. ${ }^{76}$

\section{Conclusions}

The global health crisis of the COVID-19 pandemic has made researchers consider repurposing old drugs, such as CQ, HCQ, AZM, lopinavir, and ritonavir. Current 
literature describing the diverse mechanisms of action $C Q$ and HCQ makes these drugs favorable candidates for pre- and post-infection use. However, several studies have reported no benefit with these drugs, and fatal adverse events with $C Q$ and $H C Q$ have resulted in the issuance of precautionary measures when recommending these drugs in COVID-19 patients. Larger randomized, dose determining, controlled clinical trials must be done before making any final recommendation for CQ and HCQ use in COVID-19.

Article citation: Ghose M, Patel M, Nugent K. Hydroxychloroquine: A review of its effects on viral replication based on current literature. The Southwest Respiratory and Critical Care Chronicles 2020;8(36):47-54 From: Department of Internal Medicine, Texas Tech University Health Sciences Center, Lubbock, Texas

Submitted: 9/11/2020

Accepted: 10/14/2020

Reviewer: Jacob Nichols MD

Conflicts of interest: none

This work is licensed under a Creative Commons

Attribution-ShareAlike 4.0 International License.

\section{REFERENCES}

1. Smith CD, Cyr M. The history of lupus erythematosus. From Hippocrates to Osler. Rheum Dis Clin North Am 1988;14(1): $1-14$.

2. Page F. Treatment of lupus erythematosus with mepacrine. Lancet 1951;2(6687):755-758.

3. Molad Y, Gorshtein A, Wysenbeek AJ, et al. Protective effect of hydroxychloroquine in systemic lupus erythematosus. Prospective long-term study of an Israeli cohort. Lupus 2002; 11(6):356-361.

4. Petri M. Thrombosis and systemic lupus erythematosus: the Hopkins Lupus Cohort perspective. Scand J Rheumatol 1996;25(4):191-193.

5. Clark P, Casas E, Tugwell P, et al. Hydroxychloroquine compared with placebo in rheumatoid arthritis. A randomized controlled trial. Ann Intern Med 1993;119(11):1067-1071.

6. Fox RI, Dixon R, Guarrasi V, et al. Treatment of primary Sjögren's syndrome with hydroxychloroquine: a retrospective, open-label study. Lupus 1996;5 Suppl 1: S31-S36.

7. Plantone D, Koudriavtseva T. Current and future use of chloroquine and hydroxychloroquine in infectious, immune, neoplastic, and neurological diseases: a mini-review. Clin Drug Investig 2018;38(8):653-671.

8. Jiang PD, Zhao YL, Shi W, et al. Cell growth inhibition, G2/M cell cycle arrest, and apoptosis induced by chloroquine in human breast cancer cell line Bcap-37. Cell Physiol Biochem 2008;22(5-6):431-440.

9. Zheng Y, Zhao YL, Deng X, et al. Chloroquine inhibits colon cancer cell growth in vitro and tumor growth in vivo via induction of apoptosis. Cancer Invest 2009;27(3):286-292.

10. Fan C, Wang W, Zhao B, et al. Chloroquine inhibits cell growth and induces cell death in A549 lung cancer cells. Bioorg Med Chem 2006;14(9):3218-3222.

11. Sotelo J, Briceño E, López-González MA. Adding chloroquine to conventional treatment for glioblastoma multiforme: a randomized, double-blind, placebo-controlled trial. Ann Intern Med 2006;144(5):337-343.

12. WHO Coronavirus Disease (COVID-19) Dashboard. Updated September 8, 2020 Accessed: September 9,2020. Available from: https://covid19.who.int/

13. Huang C, Wang Y, Li X, et al. Clinical features of patients infected with 2019 novel coronavirus in Wuhan, China [published correction appears in Lancet. 2020 Jan 30]. Lancet 2020;395(10223):497-506.

14. Calabrese F, Pezzuto F, Fortarezza F, et al. Pulmonary pathology and COVID-19: lessons from autopsy. The experience of European pulmonary pathologists. Virchows Arch 2020;477(3):359-372.

15. Menter T, Haslbauer JD, Nienhold R, et al. Postmortem examination of COVID-19 patients reveals diffuse alveolar damage with severe capillary congestion and variegated findings in lungs and other organs suggesting vascular dysfunction [published online ahead of print, 2020 May 4]. Histopathology. 2020;77(2):198-209.

16. Zhu FC, Guan XH, Li YH, et al. Immunogenicity and safety of a recombinant adenovirus type-5-vectored COVID-19 vaccine in healthy adults aged 18 years or older: a randomised, double-blind, placebo-controlled, phase 2 trial. Lancet 2020; 396(10249):479-488.

17. Savarino A, Boelaert JR, Cassone A, et al. Effects of chloroquine on viral infections: an old drug against today's diseases? Lancet Infect Dis 2003;3(11):722-727.

18. Martin RE, Marchetti RV, Cowan AI, et al. Chloroquine transport via the malaria parasite's chloroquine resistance transporter. Science 2009;325(5948):1680-1682.

19. Kaufmann AM, Krise JP. Lysosomal sequestration of aminecontaining drugs: analysis and therapeutic implications. J Pharm Sci 2007;96(4):729-746.

20. Lotteau V, Teyton L, Peleraux A, et al. Intracellular transport of class II MHC molecules directed by invariant chain. Nature 1990;348(6302):600-605. 
21. Wu SF, Chang CB, Hsu JM, et al. Hydroxychloroquine inhibits CD154 expression in CD4+ T lymphocytes of systemic lupus erythematosus through NFAT, but not STAT5, signaling. Arthritis Res Ther 2017;19(1):183.

22. Kuznik A, Bencina M, Svajger U, et al. Mechanism of endosomal TLR inhibition by antimalarial drugs and imidazoquinolines. J Immunol 2011;186(8):4794-4804.

23. An J, Woodward JJ, Sasaki T, et al. Cutting edge: Antimalarial drugs inhibit IFN-production through blockade of cyclic GMP-AMP synthase-DNA interaction. J Immunol 2015; 194(9):4089-4093.

24. van den Borne BE, Dijkmans BA, de Rooij HH, et al. Chloroquine and hydroxychloroquine equally affect tumor necrosis factor-alpha, interleukin 6 , and interferon-gamma production by peripheral blood mononuclear cells. J Rheumatol 1997;24(1):55-60.

25. Bishop NE. Examination of potential inhibitors of hepatitis A virus uncoating. Intervirology 1998;41(6):261-271.

26. Ferreira DF, Santo MPE, Rebello MA, et al. Weak bases affect late stages of Mayaro virus replication cycle in vertebrate cells. J Med Microbiol 2000;49(4):313-318.

27. Tsai WP, Nara PL, Kung HF, et al. Inhibition of human immunodeficiency virus infectivity by chloroquine. AIDS Res Hum Retroviruses 1990;6(4):481-489.

28. Randolph VB, Winkler G, Stollar V. Acidotropic amines inhibit proteolytic processing of flavivirus prM protein. Virology 1990;174(2):450-458.

29. Kronenberger P, Vrijsen R, Boeyé A. Chloroquine induces empty capsid formation during poliovirus eclipse. J Virol 1991;65(12):7008-7011.

30. Tsiang H, Superti F. Ammonium chloride and chloroquine inhibit rabies virus infection in neuroblastoma cells. Brief report. Arch Virol 1984;81(3-4):377-382.

31. Mizui T, Yamashina S, Tanida I, et al. Inhibition of hepatitis $\mathrm{C}$ virus replication by chloroquine targeting virus-associated autophagy. J Gastroenterol 2010;45(2):195-203.

32. Miller DK, Lenard J. Antihistaminics, local anesthetics, and other amines as antiviral agents. Proc Natl Acad Sci U S A 1981;78(6):3605-3609.

33. Shibata M, Aoki H, Tsurumi T, et al. Mechanism of uncoating of influenza B virus in MDCK cells: action of chloroquine. J Gen Virol 1983;64(Pt 5):1149-1156.

34. Ooi EE, Chew JS, Loh JP, et al. In vitro inhibition of human influenza A virus replication by chloroquine. Virol J 2006; 3:39.

35. Paton NI, Lee L, Xu Y, et al. Chloroquine for influenza prevention: a randomised, double-blind, placebo controlled trial [published correction appears in Lancet Infect Dis 2011 Sep;11(9):655. Smith, Annelies Wilder [corrected to WilderSmith, Annelies]]. Lancet Infect Dis 2011;11(9):677-683.
36. Yan Y, Zou Z, Sun Y, et al. Anti-malaria drug chloroquine is highly effective in treating avian influenza A H5N1 virus infection in an animal model. Cell Res 2013;23(2): 300-302.

37. Khan M, Santhosh SR, Tiwari M, et al. Assessment of in vitro prophylactic and therapeutic efficacy of chloroquine against Chikungunya virus in Vero cells. J Med Virol 2010; 82(5):817-824.

38. Randolph VB, Winkler G, Stollar V. Acidotropic amines inhibit proteolytic processing of flavivirus prM protein. Virology 1990;174(2):450-458.

39. Delvecchio R, Higa LM, Pezzuto P, et al. Chloroquine, an endocytosis blocking agent, inhibits zika virus infection in different cell models. Viruses 2016;8(12):322.

40. Glushakova SE, Lukashevich IS. Early events in arenavirus replication are sensitive to lysosomotropic compounds. Arch Virol 1989;104(1-2):157-161.

41. Porotto M, Orefice G, Yokoyama CC, et al. Simulating henipavirus multicycle replication in a screening assay leads to identification of a promising candidate for therapy. J Virol 2009;83(10):5148-5155.

42. Freiberg AN, Worthy MN, Lee B, et al. Combined chloroquine and ribavirin treatment does not prevent death in a hamster model of Nipah and Hendra virus infection. J Gen Virol 2010;91(Pt 3):765-772.

43. Dowall SD, Bosworth A, Watson R, et al. Chloroquine inhibited Ebola virus replication in vitro but failed to protect against infection and disease in the in vivo guinea pig model. J Gen Virol 2015;96(12):3484-3492.

44. Ferraris O, Moroso M, Pernet O, et al. Evaluation of CrimeanCongo hemorrhagic fever virus in vitro inhibition by chloroquine and chlorpromazine, two FDA approved molecules. Antiviral Res 2015;118:75-81.

45. Koyama AH, Uchida $\mathrm{T}$. Inhibition of multiplication of herpes simplex virus type 1 by ammonium chloride and chloroquine. Virology 1984;138(2):332-335.

46. Kouroumalis EA, Koskinas J. Treatment of chronic active hepatitis B (CAH B) with chloroquine: a preliminary report. Ann Acad Med Singap 1986;15(2):149-152.

47. Devaux CA, Rolain JM, Colson P, et al. New insights on the antiviral effects of chloroquine against coronavirus: what to expect for COVID-19? Int J Antimicrob Agents 2020;55(5): 105938.

48. Picot S, Peyron F, Donadille A, et al. Chloroquine-induced inhibition of the production of TNF, but not of IL- 6 , is affected by disruption of iron metabolism. Immunology 1993;80(1): 127-133.

49. Bondeson J, Sundler R. Antimalarial drugs inhibit phospholipase A2 activation and induction of interleukin 1beta and tumor necrosis factor alpha in macrophages: implications 
for their mode of action in rheumatoid arthritis. Gen Pharmacol 1998;30(3):357-366.

50. Jeong JY, Jue DM. Chloroquine inhibits processing of tumor necrosis factor in lipopolysaccharide-stimulated RAW 264.7 macrophages. J Immunol 1997;158(10):4901-4907.

51. Weber SM, Levitz SM. Chloroquine interferes with lipopolysaccharide-induced TNF-alpha gene expression by a nonlysosomotropic mechanism. J Immunol 2000;165(3):1534-1540.

52. Lu H. Drug treatment options for the 2019-new coronavirus (2019-nCoV). Biosci Trends 2020;14(1):69-71.

53. Mauthe $M$, Orhon $I$, Rocchi $C$, et al. Chloroquine inhibits autophagic flux by decreasing autophagosome-lysosome fusion. Autophagy 2018;14(8):1435-1455.

54. Fantini J, Di Scala C, Chahinian H, et al. Structural and molecular modelling studies reveal a new mechanism of action of chloroquine and hydroxychloroquine against SARS-CoV-2 infection. Int J Antimicrob Agents 2020;55(5):105960.

55. Matsuyama $S$, Nao N, Shirato $K$, et al. Enhanced isolation of SARS-CoV-2 by TMPRSS2-expressing cells. Proc Natl Acad Sci U S A 2020;117(13):7001-7003.

56. Hoffmann $M$, Mösbauer $K$, Hofmann-Winkler $H$, et al. Chloroquine does not inhibit infection of human lung cells with SARS-CoV-2. Nature 585, 588-590 (2020).

57. Gautret P, Lagier JC, Parola P, et al. Hydroxychloroquine and azithromycin as a treatment of COVID-19: results of an open-label non-randomized clinical trial. Int J Antimicrob Agents 2020;56(1):105949.

58. Huang M, Tang T, Pang $P$, et al. Treating COVID-19 with chloroquine. J Mol Cell Biol 2020;12(4):322-325.

59. Gao J, Tian Z, Yang X. Biosci Trends 2020;14(1):72-73.

60. Rana DR, Dulal S. Therapeutic application of chloroquine and hydroxychloroquine in clinical trials for COVID-19: a systematic review. medRxiv 2020.

61. Magagnoli J, Narendran S, Pereira F, et al. Outcomes of hydroxychloroquine usage in united states veterans hospitalized with COVID-19 [published online ahead of print, 2020 Jun 5]. Med (N Y) 2020; 10.1016/j.medj.2020.06.001.

62. Garcia-Cremades M, Solans BP, Hughes E, et al. Optimizing hydroxychloroquine dosing for patients with covid-19: an integrative modeling approach for effective drug repurposing. Clin Pharmacol Ther 2020;108(2):253-263.

63. Arshad S, Kilgore P, Chaudhry ZS, et al. Treatment with hydroxychloroquine, azithromycin, and combination in patients hospitalized with COVID-19. Int J Infect Dis 2020; 97:396-403.

64. Boulware DR, Pullen MF, Bangdiwala AS, et al. A randomized trial of hydroxychloroquine as postexposure prophylaxis for Covid-19. N Engl J Med 2020 Aug 6;383(6):517-525. doi: 10.1056/NEJMoa2016638. Epub 2020 Jun 3.
65. Mitjà $\mathrm{O}$, Corbacho-Monné $\mathrm{M}$, Ubals $\mathrm{M}$, et al. Hydroxychloroquine for early treatment of adults with mild Covid-19: A Randomized-Controlled Trial. Clin Infect Dis. 2020 Jul 16: ciaa1009. doi: 10.1093/cid/ciaa1009

66. Cavalcanti AB, Zampieri FG, Rosa RG, et al. Hydroxychloroquine with or without azithromycin in mild-tomoderate Covid-19. N Engl J Med 2020 Jul 23; doi: 10.1056/ NEJMoa2019014. Online ahead of print.

67. Pathak SK, Salunke AA, Thivari P, et al. No benefit of hydroxychloroquine in COVID-19: results of systematic review and meta-analysis of randomized controlled trials. Diabetes Metab Syndr. 2020 November-December; 14(6):1673-1680.

68. Tleyjeh I, Kashour Z, AlDosary O. The cardiac toxicity of chloroquine or hydroxychloroquine in COVID-19 patients: a systematic review and meta-regression analysis. medRxiv. 2020;2020:2016. 2006.20132878.

69. Chorin E, Dai M, Shulman E, et al. The QT interval in patients with SARS-CoV-2 infection treated with hydroxychloroquine/azithromycin. medRxiv 2020.

70. Srinivasa A, Tosounidou S, Gordon C. Increased incidence of gastrointestinal side effects in patients taking hydroxychloroquine: a brand-related issue? J Rheumatol 2017;44(3):398.

71. Gevers S, Kwa MSG, Wijnans E, et al. Safety considerations for chloroquine and hydroxychloroquine in the treatment of COVID-19. Clin Microbiol Infect 2020;26(9):1276-1277.

72. Taylor WR, White NJ. Antimalarial drug toxicity: a review. Drug Saf 2004;27(1):25-61.

73. Department of Health and Human Services Public Health Service Food and Drug Administration Center for Drug Evaluation and Research Office of Surveillance and Epidemiology. Published: May 19, 2020, Accessed: September 9, 2020 Available from: https://www.accessdata.fda.gov/drugsatfda_docs/nda/2020/OSE\%20Review_HydroxychloroquineCholorquine\%20-\%2019May2020_Redacted.pdf

74. Schrezenmeier E, Dörner T. Mechanisms of action of hydroxychloroquine and chloroquine: implications for rheumatology. Nat Rev Rheumatol 2020;16(3):155-166.

75. Lisney AR, Szelinski F, Reiter K, et al. High maternal expression of SIGLEC1 on monocytes as a surrogate marker of a type I interferon signature is a risk factor for the development of autoimmune congenital heart block. Ann Rheum Dis 2017;76(8):1476-1480.

76. FDA cautions against use of hydroxychloroquine or chloroquine for COVID-19 outside of the hospital setting or a clinical trial due to risk of heart rhythm problems. Updated; July 1, 2020, Accessed: September 9,2020. Available from: https://www.fda.gov/drugs/drug-safety-and-availability/fdacautions-against-use-hydroxychloroquine-or-chloroquinecovid-19-outside-hospital-setting-or 